\title{
PROPOSTA DE UM MODELO PARA DESAGREGAR PROJEÇÕES DEMOGRÁFICAS DE GRANDES ÁREAS EM SEUS COMPONȨNTES GEOGRÁFICOS*
}

\author{
Célia Landmann Szwarcwald** \\ Euclides Ayres de Castilho**
}

\begin{abstract}
SZWARCWALD, C.L. \& CASTILHO, E.A. Proposta de um modelo para desagregar projeções demográficas de grandes áreas em seus componentes geográficos. Rev. Saúde públ., S. Paulo, 23:269-76, 1989.
\end{abstract}

RESUMO: Com o objetivo de tabular informaçôes de mortalidade no Estado do Rio de Janeiro (Brasil), segundo município, e construir indicadores regionais (municipais), foi estudada a questão metodológica da estimativa dos denominadores; ou seja, de procedimentos para estimar populaçð̃es de subáreas geográficas em anos posteriores ao censo de 1980. No Brasil, as estimativas oficiais de populaçōes de subdivisões geográficas são feitas pela Fundação Instituto Brasileiro de Geografia e Estatística pelo método localmente conhecido como "método A B.". Analisa-se este procedimento e propõe-se um modelo matémático alternativo que satisfaz a condição de fechamento, isto é, a soma das alternativas para as áreas menores é igual a estimativa da população total. Uma aplicação do modelo foi feita para projetar as populaçð̃es dos municípios do Estado do Rio de Janeiro, de 1981 a 1990.

DESCRITORES: Estimativas de população, métodos. Projeções de população, métodos.

\section{INTRODUÇÃO}

Subordinados aos propósitos de tabular informaçōes de mortalidade no Estado do Rio de Janeiro desagregadas por município, defrontamo-nos com o problema teórico da estimativa dos denominadores, ou seja, da projeção de tamanhos de populações de subáreas geográficas em anos posteriores ao censo de 1980.

Diferentes métodos são aplicados, em geral, a estimativas de populações de áreas menores, como sub-regiões de um estado, municípios e distritos. Mesmo em países que contam com bom sistema de registro civil, onde as estimativas anuais de crescimento para anos posteriores ao censo são feitas pelo método direto de componentes $^{9}$ (pelas estatísticas de nascimentos, mortes e migração), a menor disponibilidade de dados locais, em particular registros de migração interna, apresenta-se como problema a requerer procedimentos não usuais.

Alguns métodos foram propostos para eștimar o componente local de migração interna, baseados em séries de dados locais administrativos, sob a suposição que os dados de migração interna são relacionados com determinado segmento da população?.
Ainda envolvendo o uso de uma ou mais séries de dados locais (tais como ocupação das escolas, número de instalaçðes elétricas, de gás ou água, auto-registro, estatísticas de nascimento e morte), outros métodos são também utilizados para estimar diretamente as populações das subáreas, ao invés de meramente o componente de migração? .

Em países onde há ausência ou deficiências na cobertura de dados locais em subáreas geográficas, recorre-se ao uso de modelos matemáticos, apesar de reconhecidamente mais imprecisos ${ }^{11}$. As funçōes mais comumente empregadas para este propósito são retas e exponenciais, apesar de que outras curvas também sejam ocasionalmente utilizadas, como a logística ou polinômios de maior grau.

A princípio, todos os modelos matemáticos devem estar sujeitos a restrição de fechamento, isto é, a soma das estimativas das áreas menores tem que ser igual ao resultado obtido para a estimativa da população total. Quanto à interpolação linear, esta é usada não somente para a população mas também para os seus segmentos cuja propriedade de fechamento é sempre satisfeita. No entanto, sabe-se que a projeção linear só deve ser utilizada quando existe razão de se

\footnotetext{
* Parte do projeto "Perfil de Mortalidade no Estado do Rio de Janeiro no período de 1976 a 1984, financiado pela Financiadora de Estudos e Projetos (FINEP) (Processo 43.86.0020.00/40).

** Centro de Informaçðes para a Saúde/Fundação Oswaldo Cruz - Av. Brasil, 4.365 - 21040 - Rio de Janeiro, RJ Brasil.
} 
acreditar que o tamanho da população varia uniformemente no período considerado.

No que se refere à projeção geométrica, geralmente são encontradas taxas de crescimento para as subpopulações diferentes da obtida para a população total. Por esta razão, a interpolação geométrica não satisfaz a restrição de fechamento e um ajuste faz-se necessário sempre que este método é empregado.

No Brasil, tanto as estimativas oficiais de populações de subdivisões geográficas como as estimativas de todos os municípios do País, para anos terminados em 5, são feitas pela Fundação Instituto Brasileiro de Geografia e Estatística (FIBGE) com um método localmente conhecido como o "método $A_{1} B_{1}$ " ". Amplamente divulgado por Madeira', este método objetiva estimar populações de áreas menores, com o compromisso de fechamento, sob a suposição que os tamanhos das subpopulações são uma função linear da estimativa da população total.

No presente trabalho, analisa-se o chamado método $A_{1} B_{1}$ e propõe-se um modelo matemático alternativo para calcular "subprojeções", ou seja, estimar populações de sub-regiões que totalizem em cada ano o valor estimado para a população total.

Duas formas do modelo geral foram desenvolvidas e aplicadas à projeção das populações das meso-regiões do Estado do Rio de Janeiro para o ano de 1980, utilizando-se os dados dos censos de 1960 e 1970 . A comparação dos modelos propostos com o método $\mathrm{A}_{1} \mathrm{~B}_{1}$ foi feita pelo confronto entre os valores projetados e aqueles observados no censo de 1980 .

O modelo de melhor adequação foi então aplicado para projetar as populações dos municípios do Estado do Rio de Janeiro, de 1981 a 1990.

\section{MÉTODO $A_{1} B_{1}$}

Este método parte da pressuposição de que a população de cada subárea $i$ - denotada por $\mathrm{P}_{1}(\mathrm{t})$ - é expressa como uma regressão linear da subpopulação $\mathrm{P}(\mathrm{t})$, para todo tempo $t$. Matematicamente:

$$
P_{1}(t)=A_{1} P(t)+B_{1}
$$

Se $t_{0}$ e $t_{1}$ correspondem a dois anos censitários, a solução é dada por:

$$
\begin{gathered}
A_{i}=\left(P_{i}\left(t_{1}\right)-P_{i}\left(t_{0}\right)\right) /\left(P\left(t_{1}\right)-P\left(t_{0}\right)\right) \\
B_{i}=P_{1}\left(t_{0}\right)-A_{i} P\left(t_{0}\right)
\end{gathered}
$$

Obviamente, como $\sum_{\mathrm{i}} \mathrm{A}_{1}=1$ e $\sum_{\mathrm{i}} \mathrm{B}_{\mathrm{i}}=0 \mathrm{a}$ propriedade de fechamento é sempre cumprida.

Um problema que surge, além de outros, da utilização do método $A_{i} B_{i}$, é que o modelo de crescimento da subpopulação fica determinado pelo da população total. Assim é que quando a interpolação linear é usada para a população total, as subpopulações são conseqüentemente projetadas linearmente, o que nem sempre é uma suposição apropriada. Vale reconhecer, contudo, que este procedimento carrega consigo a vantagem de independência na seqüência de desagregações sucessivas.

Quando se supõe que a população total tem um modelo geométrico de crescimento,

$\mathrm{P}(\mathrm{t})=\mathrm{P}_{0} \mathrm{e}^{\lambda_{1}}$, onde $\lambda$ é a taxa de crescimento, então:

$$
\begin{gathered}
P_{1}(t)=A_{1} P(t)+B_{1} \Rightarrow \frac{d P_{1}}{d t}=A_{1} \frac{d P}{d t} \Rightarrow \\
\begin{array}{r}
\Rightarrow \frac{d P_{1}}{d t}=A_{1} \lambda P(t) \Rightarrow \frac{d P_{1}}{d t}=\lambda\left(P_{1}(t)-B_{1}\right)= \\
=\lambda P_{1}(t)-\lambda B_{1}
\end{array}
\end{gathered}
$$

Ou seja, a taxa de crescimento da subpopulação $P_{1}$ não é proporcional ao seu tamanho, argumento que, em geral, é inválido.

\section{MODELO PROPOSTO}

O procedimento ora proposto tem o objetivo de projetar populações de subáreas em anos posteriores ao censo demográfico, com a restrição de que as subpopulações devem totalizar a população de toda a área.

Para sua proposição, consideremos conhecidas as populaçð̄es de $k$ subáreas geográficas nos anos censitários $t_{0}$ e $t_{0}+10$ e definamos:

$$
\begin{aligned}
P_{1}(t)= & \text { população da subárea } i \text { no ano } t_{0}+t, \\
& t \geqslant 0 . i=1, \ldots, k
\end{aligned}
$$

$P(t)=$ população da área total no ano $t_{0}+t$, $t \geqslant 0$.

\footnotetext{
* LINS, 1.B. - Um método que estima população para áreas menores - uma aplicação prática ao caso brasileiro. [Dados inéditos].
} 
A restrição é traduzida matematicamente por:

(1) $\sum_{j} P_{i}(t)=P(t)$, qualquer que seja $t \geqslant 0$.

O modelo geral de crescimento de uma população é dado por uma equação diferencial do tipo:

$\frac{\mathrm{dN}}{\mathrm{dt}}=\mathrm{F}(\mathrm{N}(\mathrm{t})) \cdot \mathrm{N}(\mathrm{t})$, onde $\mathrm{N}(\mathrm{t})$ é a população no tempo $t$.

Em geral a função F é considerada constante quando obtemos o modelo de crescimento geométrico ou é uma função linear decrescente de $N$, correspondente ao modelo logístico de crescimento.

Consideremos que o modelo de crescimento para a população da área toda $\mathrm{P}(\mathrm{t})$ é definido pela equação diferencial:

(2) $\frac{\mathrm{dP}}{\mathrm{dt}}=\phi(\mathrm{P}(\mathrm{t})) \cdot \mathrm{P}(\mathrm{t})$, sendo a função $\phi$ supostamente conhecida.

O valor do incremento anual na população total, dado por

$$
\text { (3) } \Delta P_{1}=P(t+1)-P(t)
$$

é estabelecido através do modelo (2) e de constantes calculadas a partir de observações nos dois anos censitários $t_{0}$ e $t_{0}+10$.

Da mesma forma, a partir da proposição de uma curva teórica $\hat{P}_{1}(t)$ de crescimento para a população da subárea $i$.

(4) $\frac{\mathrm{d} \hat{\mathrm{P}}_{1}}{\mathrm{dt}}=\phi_{1}\left(\hat{\mathrm{P}}_{1}(\mathrm{t})\right) \cdot \hat{\mathrm{P}}_{1}(\mathrm{t}), \mathrm{i}=1, \ldots, \mathrm{k}$

onde os parâmetros são estimados através de observações feitas em anos censitários, os valores de $F_{1,1}$ assim definidos:

(5) $F_{1,1}=\frac{\hat{P}_{1}(t+1)-\hat{P}_{1}(t)}{\hat{P}_{1}(t)}$ ficam determinados para todo $i$ de 1 até $k$.

Para satisfazer a condição de fechamento (1), as estimativas $\hat{\mathbf{P}}_{i}(t)$ requerem ajustamento. Sendo assim, propõe-se que para cada ano $t_{0}+t a$ população da subárea $i$ seja obtida através da equação de diferenças:
(6) $P_{1}(t+1)-P_{1}(t)=\frac{\Delta P_{1} \cdot F_{1, t}}{\sum_{i}\left(F_{1,1} \cdot P_{1}(t)\right)} \cdot P_{1}(t)$

A demonstração que o modelo definido em (6) satisfaz a restrição (1) do problema, é feita de forma indutiva:

i) Parat $=0$

$$
P_{1}(1)-P_{1}(0)=\frac{\triangle P_{0} F_{1,0}}{\sum_{i} F_{1,0} P_{1}(0)}, P_{1}(0)
$$

Então:

$$
\begin{aligned}
& \sum_{\mathrm{i}} \mathrm{P}_{1}(1)-\sum_{\mathrm{i}} \mathrm{P}_{1}(0)=\Delta \mathrm{P}_{0} \Rightarrow \\
& \Rightarrow \quad \sum_{\mathrm{i}} \mathrm{P}_{1}(1)=\mathrm{P}(0)+\Delta \mathrm{P}_{0}=\mathrm{P}(1)
\end{aligned}
$$

ii) Suponhamos válido para t. Então:

$$
\begin{aligned}
& \sum_{\mathrm{i}} \mathrm{P}_{1}(\mathrm{t}+1)-\sum_{\mathrm{i}} \mathrm{P}_{1}(\mathrm{t})=\Delta \mathrm{P}_{1} \Rightarrow \\
& \sum_{\mathrm{i}} \mathrm{P}_{1}(\mathrm{t}+1)=\mathrm{P}(\mathrm{t})+\Delta \mathrm{P}_{1}=\mathrm{P}(\mathrm{t}+1)
\end{aligned}
$$

A utilização do modelo (6) é feita de maneira recursiva. Primeiramente, os valores de $\hat{\mathrm{P}}_{i}(1)$ $\mathrm{i}=1, \ldots, \mathrm{k}$ e $\mathrm{P}(1)$ são obtidos através dos modelos propostos respectivamente para o crescimento das subáreas i e da área total. Nota-se que os valores de $\hat{P}_{(1)}(1)$ não totalizam $\mathbf{P}(1)$. A partir das equações (3) e (5) são obtidos os valores de $\Delta \mathrm{P}_{0}$ e $\mathrm{F}_{\mathrm{i}, 0}, \mathrm{i}=1, \ldots, \mathrm{k}$ e substituindo-os no modelo (6) são encontrados os valores de $P_{1}(1)$, cuja soma é $P(1)$. Recursivamente, as estimativas de $P_{i}(2)$ são feitas através de $\Delta P_{1}, F_{1,1}$ e $P_{j}(1)$ e assim sucessivamente.

$O$ procedimento geral foi desenvolvido com dois casos de funções particulares $\phi$ e $\phi_{1}$, comumente usadas em modelos de crescimento de populações.

\section{Primeiro Caso:}

Suponhamos primeiramente modelos de crescimento geométrico tanto para população da área toda como para a subárea $i$.

Consideremos, então que:

$$
\begin{array}{ll}
P(t)=P(0) e^{r(t-10)}, r=\frac{1}{10} & \log (P(10) / P(0))^{*} \\
\hat{P}_{1}(t)=P_{i}(0) e^{r_{i}\left(t^{-}-10\right)}, r_{1}=\frac{1}{10} & \log \left(P_{i}(10) / P_{i}(0)\right)
\end{array}
$$

* Entende-se log por logaritmo neperiano. 
Então $\Delta P_{1}=P(t+1)-P(t)=q P(t)$ onde $q=e^{r}-1$ e $F_{i, t}=\frac{\hat{P}_{i}(t+1)-\hat{P}_{i}(t)}{\hat{P}_{i}(t)}=q_{1}$ onde $\mathrm{q}_{1}=\mathrm{e}^{r_{1}}-1$

Substituindo os valores de $P_{1}$ e $F_{i, 1}$ em (6), a fórmula recursiva de obtenção de $P_{1}(t)$, temos:

$$
P_{1}(t+1)-P_{1}(t)=\frac{q P(t) \cdot q_{i} P_{i}(t)}{\sum_{i} q_{1} P_{1}(t)}
$$

Obtém-se primeiramente o valor de $P_{i}(1)$ a partir de $P_{i}(0)$ :

$$
P_{i}(1)-P_{i}(0)=\frac{q P(0) \cdot q_{1} P_{1}(0)}{\sum_{i} q_{1} P_{1}(0)}
$$

O valor de $P_{i}(2)$ a partir de $P_{1}(1)$ e assim sucessivamente.

\section{Segundo Caso:}

Suponhamos agora que a população da área total cresce geometricamente (i.e. $P(t)=P(0)$ $\mathrm{e}^{r(1-0)}$ enquanto que a população de cada subárea tem um "fator inibidor" ao seu crescimento que é a população total $\mathrm{P}(\mathrm{t})$, sendo definida pela equação diferencial:

$$
\frac{\mathrm{d} \hat{\mathrm{P}}_{1}}{\mathrm{dt}}=\left(\dot{\alpha}_{1}-\beta_{1} \mathbf{P}(\mathrm{t})\right) \hat{\mathrm{P}}_{\mathrm{i}}(\mathrm{t}), \mathrm{i}=1, \ldots, \mathrm{k}
$$

Em estudos de dinâmicas de populações, este modelo representa o crescimento de espécies competitivas $^{8}$. No nosso caso, as espécies são as subpopulações competindo pelo tamanho limitado que é o da população total. O parâmetro $\alpha_{i}$ representa a taxa natural de crescimento da subpopulação $i$ caso não houvesse a presença de outros fatores, como as migrações internas.

Para obter a solução diferencial acima proposta, façamos:

$$
\frac{1}{\hat{P}_{1}(t)} \frac{d \hat{P}_{1}}{d t}=\alpha_{i}-\beta_{i} P(t)
$$

E integrando-se, obtemos:

$$
\hat{\mathbf{P}}_{1}(\mathrm{t})=\mathrm{C}_{0} \exp \left\{\alpha_{1} \mathrm{t}-\beta_{\mathrm{i}} \mathrm{r} \mathrm{P}(\mathrm{t})\right\}
$$

Para satisfazer a condição inicial $\hat{P}_{1}(t)=P_{1}(0)$ no ponto $t=t_{0}$, obtemos:

(7) $\hat{P}_{i}(t)=P_{i}(0) \exp \left\{\alpha_{1}\left(t-t_{0}\right)-\beta_{1} r(P(t)-P(0))\right\}$ que é a solução.

O parâmetro $\alpha_{1}$ pode ser estimado diretamente pela diminuição entre a taxa de natalidade e a taxa de mortalidade para cada subárea em ano censitário, já que ele representa a taxa natural de crescimento caso não houvesse a presença do "fator inibidor".

$\mathrm{O}$ parâmetro $\beta_{\mathrm{i}}$ é obtido a partir da seguinte equação:

$$
\beta_{i}=\frac{\alpha_{i}-1 / 10 \log \left(\mathrm{P}_{\mathrm{i}}(10) / \mathrm{P}_{\mathrm{i}}(0)\right)}{\mathrm{r}(\mathrm{P}(10)-\mathrm{P}(0)) / 10}
$$

Do modelo (7) deduzimos o valor de $F_{i, t}$ :

$$
\begin{aligned}
& \mathrm{F}_{\mathrm{i}, \mathrm{t}}=\frac{\hat{\mathrm{P}}_{\mathrm{i}}(\mathrm{t}+1)-\hat{\mathrm{P}}_{\mathrm{i}}(\mathrm{t})}{\hat{\mathrm{P}}_{\mathrm{i}}(\mathrm{t})}= \\
& \exp \left\{\alpha_{\mathrm{i}}-\beta_{\mathrm{i}} \mathrm{r}(\mathrm{P}(\mathrm{t}+1)-\mathrm{P}(\mathrm{t}))\right\}-1
\end{aligned}
$$

As subpopulaç̃̃es $P_{i}(t)$ são estimadas através da equação recursiva (6).

\section{ADEQUAÇÃO DOS MODELOS}

Foram testadas empiricamente as adequações de três modelos: o primeiro caso do modelo proposto, que denominamos de Modelo 1: o segundo caso de Modelo 2 e o método $A_{i} B_{i}$ de Modelo $\mathbf{A}_{\mathrm{i}} \mathbf{B}_{\mathrm{i}}$.

Para os testes foram utilizadas as populações das meso-regiões do Estado do Rio de Janeiro:

i) Fluminense do Grande Rio

ii) Industrial do Médio Paraíba

iii) Litoral Sul

iv) Baixadas Litorâneas

v) Serrana

vi) Norte

vii) Município do Rio de Janeiro

Os dados populacionais observados nos censos de 1960 e $1970^{1}$ foram utilizados para os cálculos dos parâmetros dos três modelos. As subpopulações das regiōes do Estado foram então projetadas para o ano de 1980 pelos três métodos diferentes e comparados com os valores observados no censo de $1980^{4}$ (Tabela 1). Supôs-se sempre uma taxa de crescimento anual para o conjunto do Estado de 2,25\% da década de 7080 , estimativa esta resultante da projeção linear das taxas de crescimento observadas para as décadas de 1950-60 e 1960-70.

$\mathrm{Na}$ aplicação do Modelo 2, os dados de nascimento foram obtidos pelo censo de $1970^{3}$, e corrigidos pelo fator resultante da aplicação do método de Brass ${ }^{2}$ de correção da fecundidade no Estado. Os dados de óbito regionais para o ano de 1970 foram obtidos pela publicação da Fundação Instituto de Desenvolvimento Econômico e Social (FIDERJ) ${ }^{6}$ e corrigidos conjecturando-se uma cobertura do sistema de $95 \%$. 
TABELA 1

Valores observados e projetados das populaçōes das meso-regiōes do Estado do Rio de Janeiro, 1980.

\begin{tabular}{lcccc}
\hline & $\begin{array}{c}\text { Valores Observa- } \\
\text { dos no censo de } \\
1980^{*}\end{array}$ & Modelo 1 & Modelo 2 & Modelo $\mathrm{A}_{\mathrm{i}} \mathrm{B}_{\mathrm{i}}$ \\
\cline { 3 - 5 } & 3.923 .574 & 4.053 .873 & 4.006 .506 & 3.932 .281 \\
\hline $\begin{array}{l}\text { Fluminense do } \\
\text { Grande Rio }\end{array}$ & 802.633 & 729.805 & 741.119 & 744.934 \\
$\begin{array}{l}\text { Industrial do } \\
\text { Médio Paraíba }\end{array}$ & 78.460 & 70.772 & 71.885 & 71.459 \\
$\begin{array}{l}\text { Litoral Sul } \\
\text { Baixadas Lito- } \\
\text { râneas }\end{array}$ & 390.854 & 374.334 & 382.944 & 381.513 \\
$\begin{array}{l}\text { Serrana } \\
\text { Norte }\end{array}$ & 337.482 & 306.832 & 316.398 & 313.062 \\
$\begin{array}{l}\text { Município do } \\
\text { Rio de Janeiro }\end{array}$ & 667.817 & 634.421 & 664.637 & 630.806 \\
\hline $\begin{array}{l}\text { Estado do Rio de } \\
\text { Janeiro }\end{array}$ & 5.090 .700 & 5.094 .357 & 5.080 .905 & 5.190 .339 \\
\hline
\end{tabular}

Taxa de crescimento utilizada para a população total: $r=0,0225$

* Fundação IBGE ${ }^{4}$.

O critério escolhido para avaliação do grau de discrepância entre o modelo e os dados ob. servados baseou-se na estimativa de uma regressão linear entre os valores projetados pelo modelo e os valores observados para o ano de 1980. Na suposição de valores projetados idênticos aos observados, o coeficiente angular $b$ seria igual a $l$ e o coeficiente linear $a$ seria igual a 0 .

De posse das estimativas de $\hat{a}$ e $\bar{b}$ para os três modelos, considerou-se as hipóteses de que $b=$ $1 \mathrm{e} a=0$, testadas pelas estatísticas $t$ de Student ${ }^{10}$. Os resultados estão dispostos na Tabela 2. Segundo este critério, o "melhor" foi o Modelo 2, que mostrou a melhor aceitação para as hipóteses consideradas, pelo menos no que tange aos dados do Rio de Janeiro. Seguiu-se o Modelo 1 também demonstrando aceitação. A hipótese nula $H: b=1$ foi rejeitada na aplicação do método $A_{i} B_{i}$ com valor da estatística $t$ maior que o valor crítico $(p<5 \%)$.

\section{ESTIMATIVAS DAS POPULAÇÕES DOS MUNICÍPIOS DO ESTADO DO RIO DE JANEIRO: 1981 A 1990}

Tendo sido o Modelo 2 aquele que mostrou melhor adequação aos dados observados, escolheu-se este método para projetar as popu-

TABELA 2

Valores do coeficiente angular $(\hat{b})$, do coeficiente linear $(\hat{a})$ e respectivas estatísticas $t$ da regressão linear entre os valores projetados e os observados das populações das meso-regiōes do Estado do Rio de Janeiro, 1980.

\begin{tabular}{l|cccccc}
\hline Modelo & $\hat{b}$ & $\begin{array}{c}\mathrm{t} \\
\mathbf{p a r a} \\
\mathbf{H}: \mathbf{b}=1\end{array}$ & $\hat{p}$ & $\hat{\mathbf{a}}$ & $\begin{array}{c}\mathrm{t} \\
\mathrm{para} \\
\mathrm{H}: \mathrm{a}=0\end{array}$ \\
\hline Modelo 1 & 1,019 & 1,74 & NS* & $-35.231,72$ & $-1,27$ & NS \\
Modelo 2 & 1,011 & 1,26 & NS & $-20.828,57$ & $-1,01$ & NS \\
Modelo $\mathrm{A}_{\mathrm{i}} \mathrm{B}_{\mathrm{i}}$ & 1,021 & 3,19 & $2 \%<\mathrm{p}<5 \%$ & $-36.961,29$ & $-2,33$ & NS \\
\hline
\end{tabular}

* NS = não significativo ao nível de $5 \%$. 


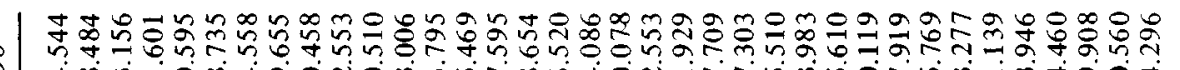

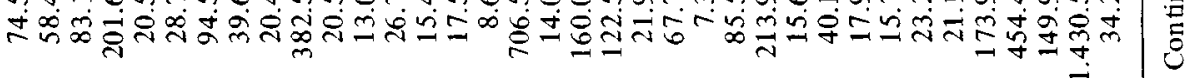

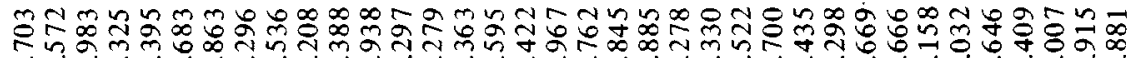

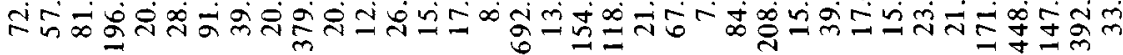

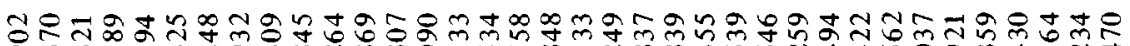

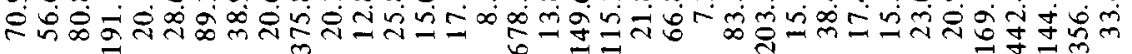

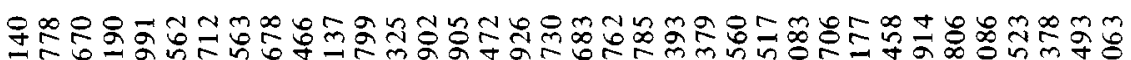

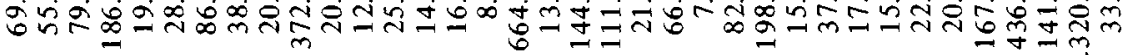

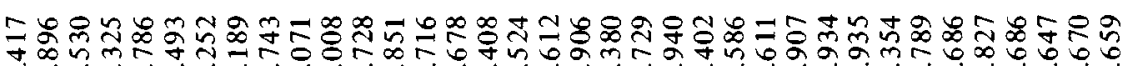

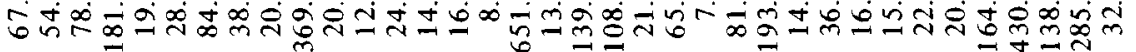

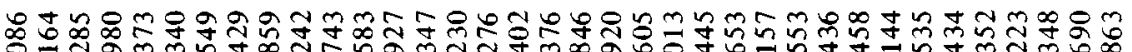

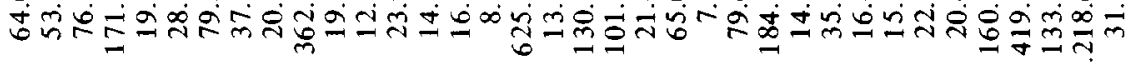

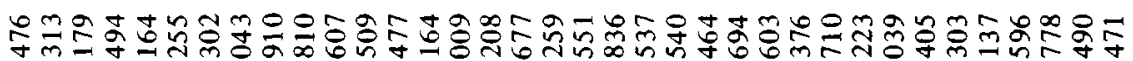

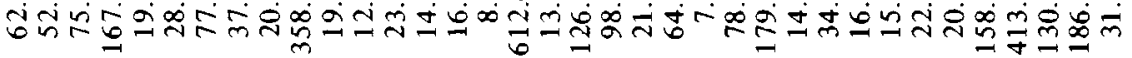

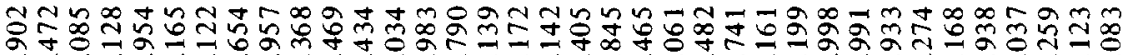

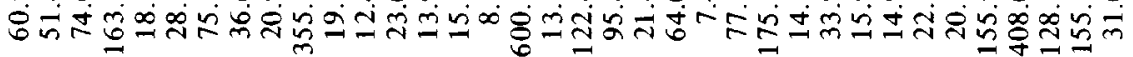

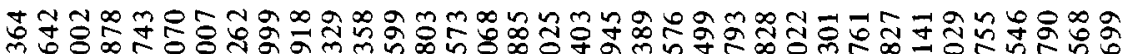

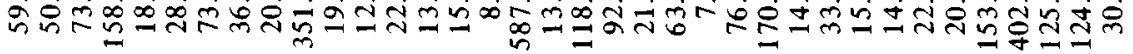

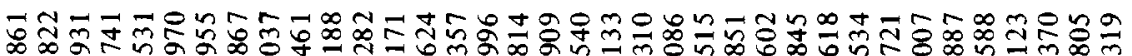

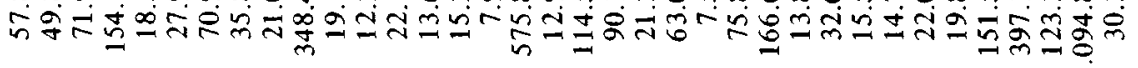

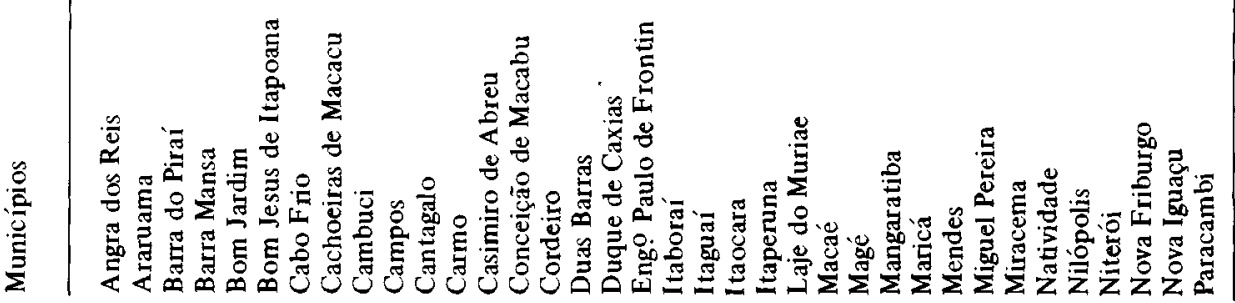




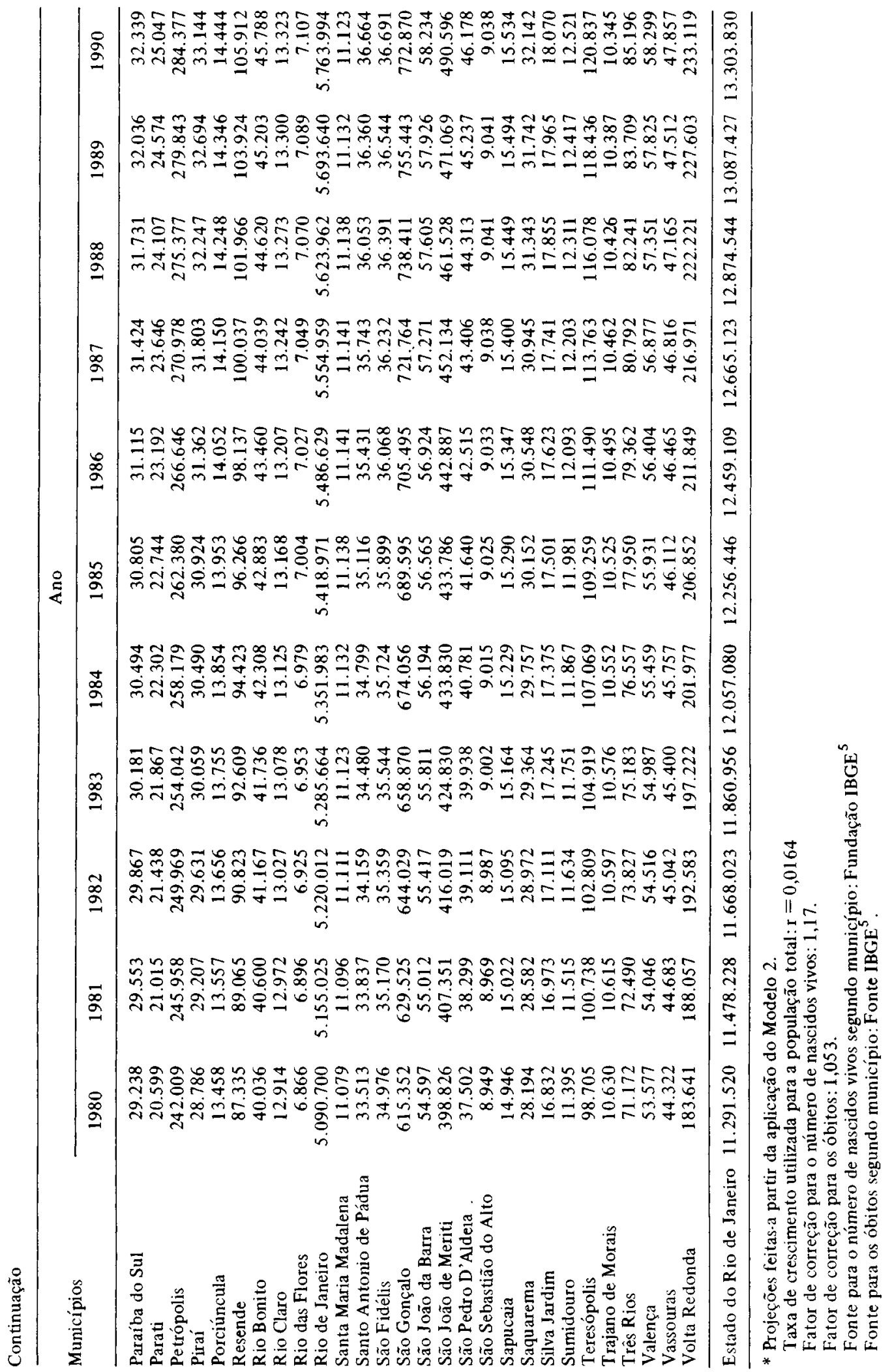


lações dos municípios do Estado do Rio de Janeiro no período de 1981 a 1990.

Foi confeccionado um aplicativo em linguagem BASIC que encontra-se à disposição no Centro de Informações para a Saúde/ Fundação Oswaldo Cruz (FIOCRUZ).

Supôs-se para a população total um modelo geométrico de crescimento, com uma taxa de crescimento de $1,64 \%$ ao ano, na década de 1980-90. Esta estimativa é a projeção das taxas de crescimento encontradas para o Estado nas décadas de 1950-60, 1960-70 e 1970-80.

Para estimativas dos parâmetros $\alpha_{i}$, as taxas de natalidade municipais foram obtidas pelo censo de $1980^{4}$ enquanto os dados de óbitos municipais, para o ano de 1980 , o foram pelas Estatísticas de Registro Civil's. Tanto o número de óbitos como o de nascidos vivos foram corrigidos segundo método proposto em trabalho anterior*.

As estimativas das populaçðes dos municípios do Estado do Rio de Janeiro, no período de 1981 a 1990, resultantes da aplicação do segundo caso do modelo proposto, encontram-se dispostas na Tabela 3. As subpopulações totalizam a cada ano a população projetada para o Estado.

\section{AGRADECIMENTOS}

Aos redatores da Revista de Saúde Pública pelas valiosas sugestões.

SZWARCWALD, C.I. \& CASTILHO, E.A. [Proposal for a model for the desaggregation of demographic projections relating to large areas into their geographical components]. Rev. Saúde públ., S. Paulo, 23:269-76, 1989.

\begin{abstract}
The problem of the preparation of estimates of the total population of geographic subdivisions after the 1980 census is studied with a view to the tabulation on mortality data of Rio de Janeiro State (Brazil) by municipal districts and the estimation of regional mortality rates. In Brazil, the calculation of the official population estimates is undertaken by the Brazilian Institute of Geography and Statistics. The method employed to estimate the population of geographic subdivisions is known as the " $A_{1} B_{1}$ method". This procedure is analysed and an alternative mathematical model is proposed that also satisfies the closure condition, that is, the projected values for the sum of the segments up to the total population projection. As an application of the proposed model, estimates of the population of the municipal districts of Rio de Janeiro State were prepared covering the period from 1981 to 1990.
\end{abstract}

KEYWORDS: Population estimates, methods. Populations projection, methods.

\title{
REFERÊNCIAS BIBLIOGRÁFICAS
}

1. ANUÁRIO ESTATÍSTICO DO ESTADO DO RIO DE JANEIRO 1981. (Fundação de Amparo à Pesquisa do Estado do Rio de Janeiro), Rio de Janeiro, 1981.

2. BRASS, W. et al. The demography of tropical Africa. Princeton, N.J., Princeton University Press, 1968.

3. FUNDAÇÃO IBGE. Censo demográfico: Rio de Janeiro. Rio de Janeiro, 1973. v.1, t.1. (VIII Recenseamento Geral do Brasil - 1970).

4. FUNDAÇĀO IBGE. Censo demográfico: Rio de Janeiro. Rio de Janeiro, 1983, v.1, t.4, n.18. (IX Recenseamento Geral do Brasil - 1980).

5. FUNDAÇÃO IBGE. Estatísticas do registro civil: 1980. Rio de Janeiro, 1981. v.7.

6. FUNDAÇÃO INSTITUTO DE DESENVOLVIMENTO ECONÔMICO E SOCIAL (FIDERJ/DIGEST/ SIPE). Estado do Rio de Janeiro: estatísticas do sistema de saúde. Rio de Janeiro, 1977.

7. MADEIRA, J.L. \& SIMŐES, C.C.S. Estimativas preliminares da população urbana e rural segundo as
Unidades da Federação de $1960 / 1980$ por uma nova metodologia. Rev. bras. Estat., Rio de Janeiro, 33(129):3-11, 1972.

8. PIELOU, E.C. An introduction to mathematical ecology. New York, John Wiley \& Sons, 1969.

9. SHRYOCK, H.S. et al. Population estimates. In: U.S. Bureau of the Census. The methods and materials of demography. $3^{\text {rd }}$ ed., Washington, D.C., U.S. Government Printing Office, 1975. p.725-70.

10. SNEDECOR, G.W. \& COCHRAN, W.G. Statistical methods. $6^{\text {th }}$ ed. Iowa, The Iowa State University Press, 1967.

11. ZITTER, M. \& SHRYOCK, H.S. Accuracy of methods of preparing postcensal estimates for states and local areas. Demography, 1(1):227-41, 1964.

Recebido para publicação em 30/6/1988. Reapresentado em 7/6/1989.

Aprovado para publicação em 8/6/1989.

* Szwarcwald, C.L. - A mortalidade infantil no Brasil de 1977 a 1985: uma proposta de método de cálculo a partir das estatísticas vitais. [Dados inéditos] 